\title{
Clinico-Radiological Outcome of Low Profile Pre-Contoured Anatomical Locking Plates Fixation in Displaced Intraarticular Calcaneum Fracture
}

\author{
Neetin P Mahajan ${ }^{1}$, Lalkar Laxman Gadod ${ }^{2 *}$, Mrugank Narvekar ${ }^{1}$, \\ Ishan Gajbe ${ }^{3}$, Swapnil Bhalerao ${ }^{3}$ and Kartik Pande ${ }^{1}$ \\ ${ }^{1}$ Department of Orthopaedics, Grant Government Medical College, Mumbai, Maha- \\ rashtra, India \\ ${ }^{2}$ Department of Orthopaedics, Columbia Asia Hospital, Pune, Maharashtra, India \\ ${ }^{3}$ Department of Orthopaedics, Kamala Nehru Hospital, Pune, Maharashtra, India \\ *Corresponding Author: Lalkar Laxman Gadod, Department of Orthopaedics, \\ Columbia Asia Hospital, Pune, Maharashtra, India.
}

Received: June 04, 2021

Published: July 07, 2021

(C) All rights are reserved by Lalkar Laxman

Gadod., et al.

\section{Abstract}

Background: Calcaneal fractures are one of the most difficult fractures to be treated by the orthopedic surgeons. Calcaneal fractures (CFs) account for more than $60 \%$ of tarsal bone fractures and around $2 \%$ of all fractures. This can be a debilitating fracture and is most common in those who are economically active.

Methods: Twenty-four patients with a history of fall from height, and having closed intra-articular joint depression type of calcaneum fractures, included in the study, after obtaining proper valid written informed consent. CT scan of the calcaneum was also done in all patients to further delineate the type and pattern of fractures, and fracture was classified by the Sanders classification system. Functional outcome was measured with the American Orthopaedic Foot and Ankle Society (AOFAS) score 10 and the Maryland Foot Score which includes pain, function and alignment. Radiological outcome was assessed by the angle of Bohler and assessing union of fracture.

Results: The mean AOFAS score at 9 months was $78.87 \pm 9.86$. According to the AOFAS score, at 9 months, $16(66.67 \%)$ patients had good outcome, $5(20.83 \%)$ patients had fair outcome and $3(12.5 \%)$ patients had poor outcome.

Conclusion: ORIF of closed type II, III and IV sanders calcaneal fractures have superior radiographic and functional outcomes ORIF has to be undertaken only when swelling and fracture blisters are completely resolved. ORIF with bone graft insertion of displaced intra-articular calcaneum fractures is an effective method for treatment as it enables anatomical reduction of fracture and articular surface, restoration of Bohler's angle, and early union by the bone graft.

Keywords: Calcaneum; Fracture; Intra-Articular; Locking Plate; Pre-Contoured

\section{Introduction}

Calcaneal fractures are one of the most difficult and challenging fractures to be treated by the orthopedic surgeons. Calcaneal fractures (CFs), most commonly seen in economically active population, comprise $2 \%$ of all fractures and more than $60 \%$ of tar- sal bone fractures encountered [1,2]. Ideal management of these fractures remains controversial. Open reduction internal fixation (ORIF), performed utilizing an expansile lateral approach, has been associated with wound-related complications or flap necrosis [3]. These fractures are the result of direct impact caused by the axial 
loading of the calcaneum, that is, fall from height, and therefore, many of these are bilateral or associated with pelvic or vertebral fractures [4,6]. About $75 \%$ of these fractures are intra-articular [4-6] and comminuted with associated osteoporosis, which makes treatment of these fractures difficult. Their management remains controversial, mainly due to a low level of evidence.

Sanders computerized tomography (CT) classification [7] and the Böhler angle measurement [8] have been proven to be useful as diagnostic tools. Though conservative management of these fractures has been advised by some authors $[9,10]$, even for displaced fractures with intra-articular extension, the outcome of these have shown poor results [11]. Intraarticular and comminuted calcaneal fractures when managed conservatively has lead to persistent pain and stiffness associated with delayed functional recovery.

Open reduction with internal fixation using the extended lateral approach, which allows better visualization of the subtalar joint, is the most widely used technique in the management of displaced intra-articular fractures. However, the use of plate fixation has been associated with post-operative complications including deep infection (8 - 25\%), superficial infection (40\%) and wound necrosis $(14 \%)$, owing to the thin soft tissue coverage over the operative area [11].

\section{Aim of the Study}

The present study aims at evaluating the radiological and functional outcome of displaced intra-articular calcaneum fractures, operated with open reduction and internal fixation (ORIF) with $2.7 \mathrm{~mm}$ anatomically contoured locking plate with bone graft.

\section{Materials and Methods}

A prospective observational study was conducted from 2015 to July 2020 at our tertiary care Centre. After getting permission of the institutional ethical committee. Twenty-four patients presenting to our tertiary care center with closed intra-articular joint depression type of calcaneum fractures with a history of fall from height were included in the study, after obtaining proper valid written informed consent.

\section{Inclusion criteria}

All patients between the ages of 20 - 75 years with closed displaced intra-articular calcaneal fracture (Sander's type II-IV).

\section{Exclusion criteria}

Patients with neurovascular deficit, open fractures, rheumatological diseases, previous foot or ankle surgery, and history of any drug abuse were excluded from the study.

Patients with paraplegia/paraparesis, long-bone fractures in ipsilateral limb, open fractures, fractures of other tarsal bones, and arthritis of the ankle or subtalar joint.

All patients at presentation had tenderness and swelling over the affected ankle with restricted range of motion (ROM). Patients were investigated with plain radiographs of the ankle in the anteroposterior, lateral and axial views. All patients were investigated with CT scan of the calcaneum to better delineate the type and pattern of fractures, and fracture was classified by the Sanders classification system [8] (Figure 3a and 3b) All patients were given primary treatment in the form of below-knee Plaster of Paris (POP) slab, analgesic, anti-inflammatory medications. Patients were given strict limb elevation over the BB splint in the ward.

All patients were posted for surgery only once swelling subsided judged by the wrinkling of the skin over the ankle. Patients were operated under spinal anesthesia in the lateral position. The tourniquet was inflated after exsanguination of the limb. A L-shaped incision on the lateral aspect was used for exposing the fracture, running parallel and just anterior to tendoachilles, curved slightly just below lateral malleolus, and then parallel to the fifth metatarsal shaft. The skin and subcutaneous tissue was raised as a single flap with sub periosteal elevation to expose the subtalar joint, calcaneum, lateral malleolus, cuboid, and lateral navicular (Figure 1a). The flap was retracted using three smooth K-wires inserted one each in the lateral malleolus, cuboid, and the navicular, and was covered with a wet mop to keep it moist during the operative procedure. Fracture edges were freshened by scooping off the callus formed and articular reduction was achieved under direct vision and confirmed under image intensifier (Figure 2a-2c). After ensuring proper restoration of the Bohler's and the Gissane's angles, the fracture was temporarily fixed using K-wires under radiographic guidance. When the reduction was satisfactory, as seen with the radiographic intensifier, final stabilization was obtained with a low-profile locking plate. and angle-stable screws of appropriate sizes. Any bony defect persistent after reduction was filled with the cortico-cancellous bone graft retrieved from iliac crest. Wound was closed in layers under vacuum drain (Figure 1c). 


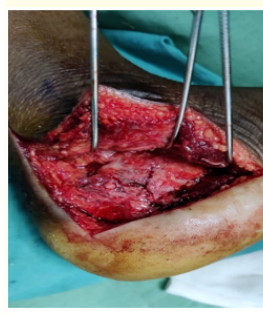

a

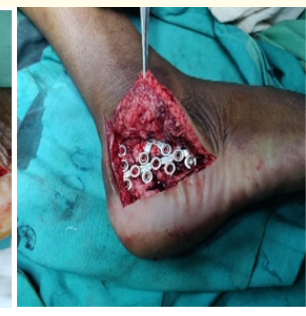

b

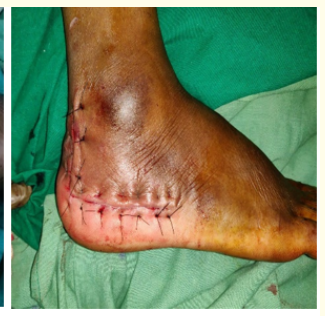

Figure 1a-1c: Interoperative pictures showing retracted flap with $\mathrm{k}$ wire fixation in figure $1 \mathrm{a}$, fracture fixation with pre-contoured locking plate in figure $1 \mathrm{~b}$, suturing in figure $1 \mathrm{c}$.

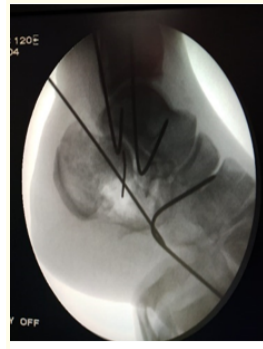

a

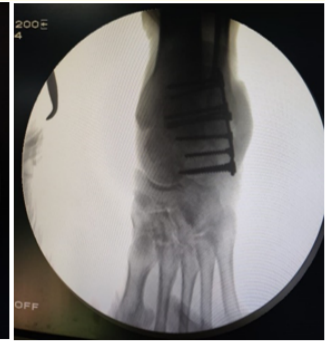

$\mathrm{b}$

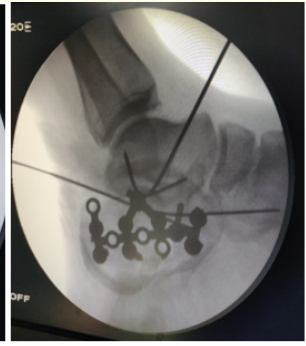

c

Figure 2a-2c: Intraoperative $\mathrm{C}$ arm images showing fracture fixation with $\mathrm{k}$ wire and locking plate.

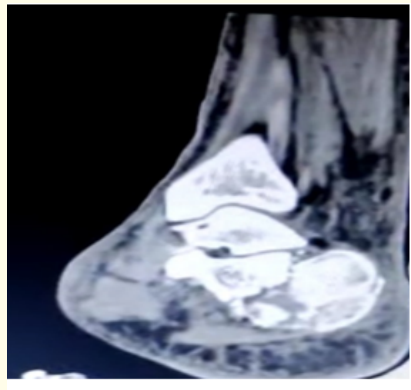

a

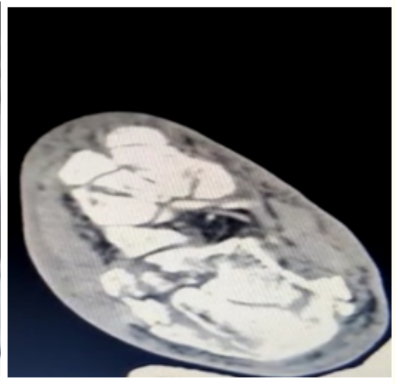

b

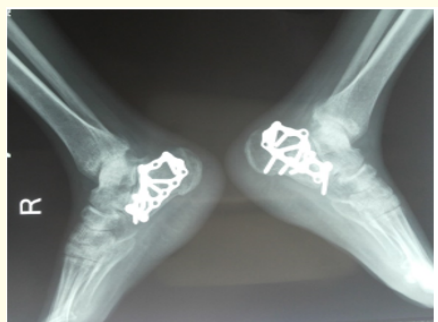

a

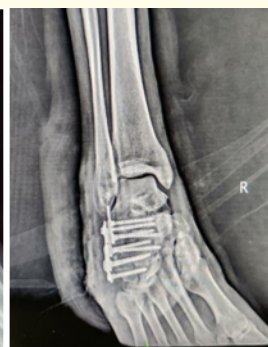

b

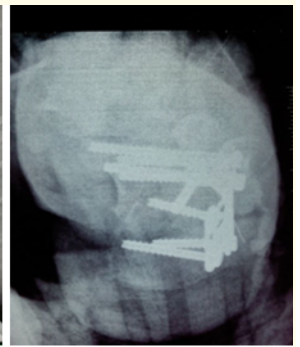

Figure 4a-4c: Immediate postoperative xrays AP, lateral and axial view.

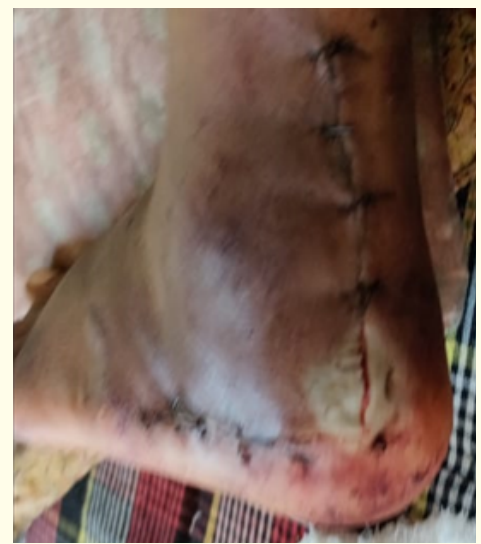

Figure 5: Showing maceration at suture site (complication).

Figure 6a and 6b: 1.5 year follow up xray showing union at fracture site. 


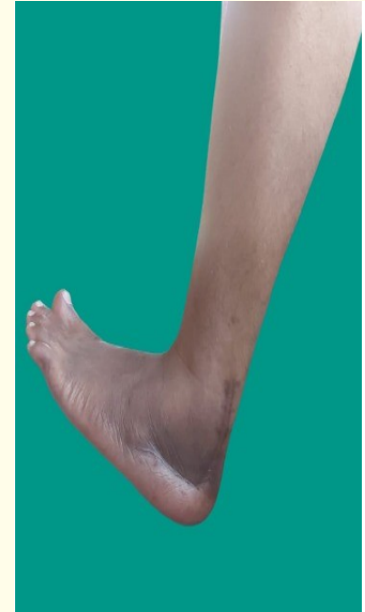

a

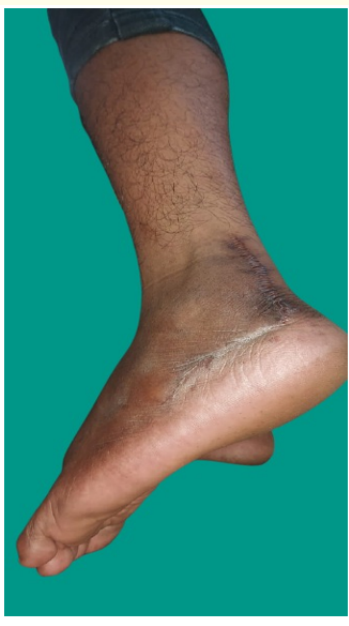

Figure 7a and 7b: Functional outcome (dorsiflexion and plantar flexion)

\section{Postoperative-care}

A compression dressing was applied on the operated side for $48 \mathrm{~h}$ after surgery. A check dress of the surgical wound was done on the $3^{\text {rd }}$ postoperative day. Patients were advised a nil-weightbearing walk over the operated limb with a walker for 8 weeks, while passive and active ankle ROM exercises were initiated 21 days post-surgery. Suture removal was delayed till 3 weeks postsurgery. Partial weight bearing over the limb was started after 8 weeks and gradually progressed to full weight bearing by 12 - 16 weeks. The patients was followed up at 6 week, 12 weeks, 6 months and 9 months.

At each follow-up, plain radiograph were assessed, and functional outcome and ankle ROM was measured. Functional outcome was measured with the American Orthopaedic Foot and Ankle Society (AOFAS) score 10 which includes pain, function, and alignment. For radiological outcome, the angle of Bohler, and progress of the union of fracture was assessed.

The radiographic evaluation comprised the analysis of conventional radiographs, including anteroposterior, lateral, axial, and internal oblique views in the preoperative, postoperative, and follow-up periods and preoperative CT scans. The fractures were clas- sified according to Essex-Lopresti [10] and Sanders., et al. [7,8,21] and the Böhler's angle was measured. The post-surgical restoration of Böhler's angle in the normal physiological range $\left(20^{\circ}-40^{\circ}\right)$ $[9,12]$ was considered a good radiological outcome.

The fracture was considered united radiologically on appearance of bony trabeculae crossing the fracture site on atleast three surfaces on orthogonal views (Figure 6a and 6b). The time taken for clinical and radiological union was noted. The fracture union was deemed as delayed if there are no clinical and radiological signs of union by 16 weeks, and non union was declared if no signs of union were seen till 24 weeks after injury.

Functional outcome was measured with the American Orthopaedic Foot and Ankle Society (AOFAS) score. The AOFAS score is a 100-point scoring system assessing the pain, function and alignment of the foot. The lower the score poorer the functional outcome.

The result was then graded as excellent, good, fair and poor as follows:

- $\quad$ Excellent - 89 to 100 points.

- $\quad$ Good - 79 to 89 points.

- $\quad$ Fair - 69 to 79 points.

- $\quad$ Poor - Less than 60 points.

\section{Statistical analysis}

All data were collected and was recorded into Microsoft excel spreadsheet 2016 and them imported to IBM Corp. Released in 2016. IBM SPSS Statistics for Windows, Version 24.0. Armonk, NY: IBM Corp. Tables were generated using custom tables option. The relevant, measurements were reported as mean, standard deviation, actual numbers and percentages. Student's t Test, Chi Square test and ANOVA were used appropriately for assessing group differences. When a two-tailed p value is less than 0.05 , the difference between groups was considered statistically significant.

\section{Results}

An observational prospective study of 24 consecutive patients having intraarticular joint depression type of calcaneal fractures 
was carried out in our tertiary care Institute. All patients were operated with open reduction, bone grafting, and fixation with 2.7 $\mathrm{mm}$ pre-contoured locking anatomical plate.

The mean age of the patients in the study was $40.79 \pm 15.32$ years with a range of 20 - 75 years. Out of the 24 patients, $18(75 \%)$ were males and 6 (25\%) were females. In the study, 15 (62.5\%) patients had a right sided fracture and $9(37.5 \%)$ patients had a left sided fracture; with 13 (54.16\%) patients having a fall from height and 11 (45.84\%) patients met with Road traffic accident. 6 $(25 \%)$ patients had an associated lumbar vertebral body fracture. Out of the 30 patients, 13 (54.16\%) had Sanders Type II fracture, 8 $(33.33 \%)$ had Sanders Type III fracture and 3 (12.5\%) patients had Sander Type IV fracture.

The mean time to clinical union of the fracture was $14.87 \pm 3.94$ weeks and radiological union was $13.83 \pm 3.96$ weeks. The mean range of motion at 9 months was as given in table 1 and figure $7 \mathrm{a}$ and $7 \mathrm{~b}$.

\begin{tabular}{|c|c|}
\hline Plantar Flexion & $32.08 \pm 5.08$ \\
\hline Dorsiflexion & $17.70 \pm 3.60$ \\
\hline Inversion & $17.70 \pm 3.60$ \\
\hline Eversion & $12.70 \pm 3.60$ \\
\hline
\end{tabular}

Table 1: Mean range of motion at 9 months.

The mean AOFAS score at 9 months was $78.87 \pm 9.86$. According to the AOFAS score, at 9 months, $16(66.67 \%)$ patients had good outcome, 5 (20.83\%) patients had fair outcome and $3(12.5 \%)$ patients had poor outcome.

Post-operative wound complications were seen in $4(16.67 \%)$ patients with 1 patient having wound dehiscence and 2 patients showed skin maceration around the surgical wound (Figure 5). Superficial wound infection was seen in 1 patient.

\section{Discussion}

The treatment of Calcaneal fractures remains a challenge due to the presence of comminution and complicated fracture pattern. Extra articular and undisplaced intraarticular fractures may treated conservatively with cast; however, for displaced intraarticular fractures operative management provides superior results as compared to conservative measures. Operative management can be done by closed pinning or screw fixation as well as open reduction and plate fixation. Though closed techniques are associated with less wound complication and better cosmesis, achieving anatomic reduction is difficult in these. Open techniques, on the other hand, permits manipulation of the fragments under direct vision and thus the ease in achieving anatomic reduction [18]. Also, a plate fixation provides a more rigid construct as compared to screw fixation $[20,21]$.

Buckley et. al. in their study concluded that operative management enabled anatomic reduction of the fracture fragments, thus giving better functional outcome than non-operative treatment [2]. Paul., et al. in their study similarly proved superiority of operative management.

Surgery for calcaneum fractures should be delayed to allow soft-tissue swelling to reduce, decreasing risk of wound problems post-surgery.

In the present study, all patients were operated using a lateral approach [16] with a low profile locking anatomical plate for fixation. The goals of fixation were to achieve anatomical reduction of articular surface, restoration of calcaneal width, decompression of sub fibular space available for peroneal tendons, and reduction of the calcaneocuboid joint in some cases [11,17]. Emphasis was given to the adequate restoration of articular surface and angles of Bohler and Gissane. Bone grafting was done to maintain the calcaneal height and width, to prevent the collapse of the articular cartilage, and to promote earlier fracture healing. Meticulous dissection of the soft tissues was ensured with raising deep subperiosteal flaps and avoiding rash handling during surgery, to prevent occurrence of post-operative wound complications.

The mean age of the patients in the present study was $40.79 \pm$ 15.32 years with the youngest being 22 years and the oldest being 78 years. The majority of the patients were males $(75 \%)$. The study by Mitchell., et al. [12] showed mean age of patients was 44 years with a male to female ratio of $2.4: 1$.

In the present study, 13 (54.16\%) patients had a history of fall from height 11 (45.84\%) patients met with Road traffic accident. 
The study by Cao., et al. had 78\% patient with history of fall from height, $18 \%$ patients had a road traffic accident and $3 \%$ patients had sports related injury.

In the present study, clinical union was seen at $14.87 \pm 3.94$ weeks and radiological union at $13.83 \pm 3.96$ weeks. Cao., et al. [18] in their study had observed solid union on radiographs at a mean of $8.2 \pm 1.1$ weeks.

The mean range of motion achieved at end of 9 months was: plantarflexion $32.08^{\circ}$, dorsiflexion $17.7^{\circ}$, inversion $17.7^{\circ}$, and eversion $12.7^{\circ}$. The observations were similar to those achieved by Besse., et al. plantar flexion: $35.7^{\circ}$, dorsiflexion: $13.7^{\circ}$, inversion: $13.6^{\circ}[20]$.

The mean AOFAS score at 9month follow up was 78.87. At the end of 9 month follow up, 16 patients had good outcome, 5 had fair outcome and 3 had poor outcome. The outcomes were similar to those found by Besse., et al. [20], Leung., et al. [14], Gaskill., et al. [17] and Cao., et al. [18] in their studies.

Postoperative wound complications were seen in 4 (16.67\%) patients with 1 patient developing superficial wound infection managed conservatively with antibiotics and dressings. The rate of complications in the present study was comparable to other studies.

The limitations in our study were the small number of cases and the shorter time of follow-up. Large multicentric trials may further show the efficacy of open reduction of calcaneal fractures and fixation with plates. Furthermore, comparison with cases who are treated conservatively may aid in better understanding of the management of these fractures.

\section{Conclusion}

ORIF of closed type II, III and IV sanders calcaneal fractures have superior radiographic and functional outcomes ORIF has to be undertaken only when swelling and fracture blisters are completely resolved. ORIF with bone graft insertion of displaced intraarticular calcaneum fractures ensures anatomical reduction of the fracture with adequate restoration of the articular surface and Bohler's angle, thus proving to be an effective method for treatment for these fractures.

\section{Bibliography}

1. Wang Q., et al. "Comparison of the outcomes of two operational methods used for the fixation of calcaneal fracture". Cell Biochemistry and Biophysics 72 (2015): 191-196.

2. Buckley R., et al. "Operative compared with nonoperative treatment of displaced intra-articular calcaneal fractures: A prospective, randomized, controlled multicenter trial". Journal of Bone and Joint Surgery American 84-A (2002): 1733-1744.

3. Folk JW., et al. "Early wound complications of operative treatment of calcaneus fractures: analysis of 190 fractures". Journal of Orthopaedic Trauma 13 (1999): 369-372.

4. Fitzgibbons TC., et al. "Fractures and dislocations of the calcaneus". In: Bucholz RW, Heckman JD, editros. Rockwood and Green's Factures in Adults. $5^{\text {th }}$ edition., Philadelphia: Lippincott Williams and Wilkins 3 (2001): 2133-2179.

5. Murphy GA. "Fractures and dislocations of foot". In: Canale ST, editror. Campbell's Operative Orthopaedics. $10^{\text {th }}$ edition., Philadelphia: Mosby Inc 4 (2003): 4231-4283.

6. Raymakers JT., et al. "Results after operative treatment of intra-articular calcaneal fractures with a minimum follow-up of 2 years". Injury 29 (1998): 593-599.

7. Sanders R., et al. "Operative treatment in 120 displaced intraarticular calcaneal fractures resulting used a prognostic computed tomography scan classification". Clinical Orthopaedics and Related Research 290 (1993): 87-95.

8. Sanders R. "Current concepts review: displaced intra-articular fractures of the calcaneus". Journal of Bone and Joint Surgery 82 (2000): 225-250.

9. Böhler L. "Diagnosis, pathology, and treatment of fractures of the oscalcis". Journal of Bone and Joint Surgery 13 (1931): 7589.

10. Kitaoka HB., et al. "Clinical rating systems for the ankle-hindfoot, midfoot, hallux, and lesser toes". Foot and Ankle International 15 (1994): 349-353. 
11. Griffin D., et al. "Operative versus non-operative treatment for closed, displaced, intra-articular fractures of the calcaneus: randomised controlled trial”. British Medical Journal 349 (2014): g4483.

12. Mitchell MJ., et al. "The epidemiology of calcaneal fractures". Foot 19.4 (2009): 197-200.

13. Biz C., et al. "Radiographic and functional outcomes after displaced intra-articular calcaneal fractures: A comparative cohort study among the traditional open technique (ORIF) and percutaneous surgical procedures (PS)". Journal of Orthopaedic Surgery and Research 11 (2016): 92.

14. Leung KS., et al. "Operative treatment of displaced intra-articular fractures of the calcaneum. Medium-term results". The Bone and Joint Journal - Bone 75 (1993): 196-201.

15. Vaclav Rak., et al. "Operative treatment of intra-articular calcaneal fractures with calcaneal plates and its complications". The Indian Journal of Orthopaedics 43 (2009): 271-280.

16. Frank MA., et al. "Calcaneal fractures: surgical exposure and fixation technique update". Current Orthopaedic Practice 22 (2011): 4-11.

17. Gaskill T., et al. "Comparison of surgical outcomes of intraarticular calcaneal fractures by age". Journal of Bone and Joint Surgery American 92 (2010): 2884-2889.

18. Cao L., et al. "Surgical treatment of calcaneal fractures of sanders type II and III by a minimally invasive technique using a locking plate". The Journal of Foot and Ankle Surgery 54 (2015): 76-81.

19. Daftary A., et al. "Fractures of the calcaneus: a review with emphasis on CT". Radiographics 25 (2005): 1215-1226.

20. Besse J-L., et al. "Calcaneal intra-articular fracture osteosynthesis: Clinical and radiological prospective study of 31 cases". Foot and Ankle Surgery 12.1 (2006): 19-27.

21. Jain V., et al. "Osteosynthesis for Intra-Articular Calcaneal Fractures". Journal of Orthopaedic Surgery 15.2 (2007): 144-148.

22. Stephenson JR. "Treatment of displaced intra-articular fractures of the calcaneus using medial and lateral approaches, internal fixation, and early motion". Journal of Bone and Joint Surgery American 69.1 (1987): 115-130.
23. Gould N. "Lateral approach to the os calcis". Foot Ankle 4.4 (1984): 218-220.

24. Saurabh Jain., et al. "Outcome of open reduction and internal fixation of intraarticular calcaneal fracture fixed with locking calcaneal plate Chinese". Journal of Traumatology 16.6 (2013): 355-360.

25. Longino D and Buckley RE. "Bone graft in operative treatment of displaced intraarticular calcaneus fractures. Is it helpful?" Journal of Orthopaedic Trauma 15.4 (2001): 280-286.

26. Leung KS., et al. "Operative treatment of intraarticular fractures of the os calcis - the role of rigid internal fixation and primary bone grafting: preliminary results". Journal of Orthopaedic Trauma 3.3 (1989): 232-240.

27. Thordarson DB., et al. "Supe- rior compressive strength of a calcaneal fracture construct augmented with remodelable cancellous bone cement". Journal of Bone and Joint Surgery American 81.2 (1999): 239-246.

28. Schildhauer TA., et al. "Open reduc- tion and augmentation of internal fixation with an injectable skeletal cement for the treatment of complex calcaneal fractures". Journal of Orthopaedic Trauma 14.5 (2001): 309-317.

29. Isaac JD., et al. "The diagnostic accuracy of Böhler's angle in fractures of the calcaneus". The Journal of Emergency Medicine 45 (2013): 879-884.

30. Hyer CF., et al. "Early weight bearing of calcaneal fractures fixated with locked plates: a radiographic review". Foot and Ankle Specialist 3 (2010): 320-323.

\section{Volume 4 Issue 8 August 2021}

(C) All rights are reserved by Lalkar Laxman Gadod., et al. 